\title{
Energy balance and body-weight stability: impact of gene-environment interactions
}

\author{
Angelo Tremblay ${ }^{1,2 *}$, Louis Pérusse ${ }^{1}$ and Claude Bouchard ${ }^{3}$ \\ ${ }^{1}$ Division of Kinesiology and \\ ${ }^{2}$ Institute of Nutraceuticals and Functional Foods, Laval University, Ste-Foy, Québec, Canada G1K 7P4 \\ ${ }^{3}$ Pennington Biomedical Research Center, Louisiana State University, Baton Rouge, LA 70808, USA
}

\begin{abstract}
Studies of monozygotic twins in the context of overfeeding and energy deficit experiments have shown that gene-environment interactions affect energy balance. From a clinical standpoint, this implies that some individuals are more susceptible to body-weight gain or loss than others because of genetic differences. This opens new perspectives in predictive medicine. In the future, health professionals should be able to count on early diagnosis of individuals at risk for developing long-term metabolic problems and obesity or for not responding adequately to clinical interventions. However, before predictive medicine is in a position to contribute significantly to prevention or treatment of patients, an enormous amount of work has to be done to identify all genetic and environmental factors of relevance, and their network of interactions.
\end{abstract}

Obesity: Genotype: Energy

Industrialization and technological advances have favoured the reduction of daily physical labour and have considerably reduced the energy expenditure necessary for daily living. This obviously has been accompanied by a greater sense of well-being and improved quality of life for millions of people. However, it has also promoted a certain number of adaptations with adverse consequences. One of these adaptations is body-fat gain that is now sufficiently pronounced to be referred to as an obesity epidemic (World Health Organization, 1998). According to the first law of thermodynamics, obesity is necessarily the consequence of a long-term imbalance between energy intake and expenditure. It is easy to simply conclude that this obesity epidemic is the result of an excess energy intake. However, in reality, it is a major challenge to identify all the factors responsible for this imbalance.

As for other biological phenotypes, it is generally accepted that energy balance components are affected by genetic and environmental factors. In this regard, it is noteworthy to emphasize that the genetic profile of most populations is quite stable and has not changed dramatically over recent decades, i.e. the period during which the obesity epidemic has emerged. On the other hand, the 20th century will appear in the history of mankind as a transition time characterized by important environmental changes influencing diet and activity habits. Moreover, modernization has also been accompanied by stress and environmental pollution that could also influence energy balance.

The apparent dominance of environmental factors to explain the obesity epidemic does not imply that genetic differences are not playing a role in the proneness of some individuals towards obesity. Indeed, there are data suggesting the existence of significant gene-environment interaction effects that make some individuals more susceptible to fat gain when exposed to an obesogenic environment. As explained later, these effects have been observed in the context of experimental overfeeding and negative energy balance studies. The main challenge is to identify the specific genes affecting the response to energy balance fluctuations.

\section{Gene-diet interaction effect}

Our first attempt to demonstrate the presence of a genediet interaction effect was an experimental overfeeding protocol with monozygotic twins. Identical twins were selected because they made it possible to compare the response to a standardized environmental dietary change on energy balance in subjects with the same or a dissimilar genetic background. Thus, the within-pair resemblance in response could be compared with the between-pair resemblance. The calculation of the within-pair:between-pair resemblance ratio provided a quantitative indicator of the gene-overfeeding interaction effect.

In a first study, a $4 \cdot 18 \mathrm{MJ}(1000 \mathrm{kcal}) / \mathrm{d}$ overfeeding was tested in twins for $22 \mathrm{~d}$. Even though this study was performed in a metabolic ward, such a level of daily energy surplus can occur under free-living conditions if one consumes a diet with a low satiating potential while being sedentary (Tremblay et al. 1989; Lawton et al. 1993). 
As expected, the simulation of an obesogenic environment for $22 \mathrm{~d}$ suggested that there was a significant geneoverfeeding interaction effect on numerous energy balance-related phenotypes. For instance, there were greater between-pair than within-pair variances for the changes in fat mass (Poehlman et al. 1986a), resting energy expenditure (Poehlman et al. 1986b) and energy cost of standardized exercise (Tremblay et al. 1987).

In a subsequent investigation, we extended the study period to $100 \mathrm{~d}$ of overfeeding by $4.18 \mathrm{MJ}(1000 \mathrm{kcal}) / \mathrm{d}$, $6 \mathrm{~d}$ per week. This constituted a more severe environmental perturbation that represented an additional step towards the simulation of an obesogenic environment. As in the first protocol, a significant within-pair resemblance was found for the changes in fat mass (Bouchard et al. 1990), suggesting that some individuals are genetically predisposed to obesity. This effect was particularly strong for the change in abdominal fat and visceral fat deposition for which the genotype-diet interaction effects remained highly significant even after statistical adjustment for the changes in body-fat mass. This study also revealed significant interaction effects for components of resting energy expenditure (Tremblay et al. 1992).

In summary, these studies strongly suggested that the predisposition to gain body fat in response to standardized overfeeding has a genetic basis. However, since the absolute energy surplus was the same for everybody, the protocols favoured the identification of genotypeoverfeeding interaction effects on energy expenditure and nutrient partitioning as revealed by body composition changes. One can also consider that genetic differences may influence feeding behaviour per se, such as favouring the intake of foods having a reduced satiating potential. Given this, it is likely that the total genetic contribution to the response to changing energy balance conditions is greater than what was measured in these overfeeding studies.

\section{Genotype-negative energy balance interaction effect}

We used exercise training as a mean to induce a standardized energy deficit while energy intake was kept constant. As for overfeeding, we began with a study of the genotype-negative energy balance interaction effect using a $22 \mathrm{~d}$ protocol, which we extended later to a $93 \mathrm{~d}$ study. Again, we observed a significant within-twin pair resemblance in the response to negative energy balance conditions for body fatness and resting energy expenditure (Poehlman et al. 1986c, 1987; Bouchard et al. 1994; Tremblay et al. 1997). This suggests that the response to a standardized energy deficit is also potentially determined by genetic factors. However, it was not possible in these studies to distinguish between the effects of exercise $v$. those of the energy deficit.

\section{Predictive medicine: the challenge of specific characterization}

Establishing that there are significant gene-environment interaction effects favouring the development of obesity or defending body fat is only a first step. It needs to be followed by studies aimed at identifying the genes and mutations responsible for such effects. In this regard, a series of studies by Ukkola \& Bouchard (2004) has recently led to the identification of several genes that appear to play a role in the response to overfeeding.

The study of gene-environment interaction effects on adiposity requires consideration of all environmental factors that have the potential to modify energy balance. For instance, variations in physical activity participation and dietary fat-diet energy density are associated with fluctuations in body fatness. However, there are data suggesting that other factors can also promote an energy imbalance. These factors include variations in dietary carbohydrates, micronutrient intake such as $\mathrm{Ca}$, dietary spices containing active agents such as capsaicin, organochlorine pollutants, patterns of feeding behaviours, alcohol intake and probably many others.

The catalogue of candidate genes potentially related to variations in body-fat stores becomes larger every year (Chagnon et al. 2003) and is likely to increase even more in a foreseeable future. A long list of potentially interesting genes can only increase the complexity of defining relevant genotype-environment interaction effects, particularly in the context of predictive medicine. Indeed, the goal here is the ability to make an early detection of individuals at risk to develop metabolic problems later in life. Moreover, predictive medicine should also help in the identification of people who might be poor or good responders to a clinical intervention.

One candidate gene that we have explored further for its potential implications in gene-age interaction effects on adiposity gain is the glucocorticoid receptor gene. It is a gene that was clearly identified as a strong candidate in the gene-overfeeding studies. Further support for the role of this gene comes from other human studies on cortisol levels (Rosmond et al. 2000). In addition, adrenalectomy is known to reverse weight gain in almost all animal models of obesity (Romsos et al. 1987; Tokunaga et al. 1989). Cortisol exerts orexigenic and anti-thermogenic effects and is correlated with changes in hunger when obese individuals experience resistance to further body fat loss in a weight-reducing programme (Doucet et al. 2000). Finally, genetic variation at the glucocorticoid receptor gene is associated with variations in abdominal visceral fat level (Buemann et al. 1997).

On the basis of these observations, we tested whether the same glucocorticoid receptor gene polymorphism could be useful to identify subjects who are at greater risk of gaining fat with ageing. As shown in Fig. 1, the mean increase in subcutaneous fat in heterozygous for the glucocorticoid receptor gene $B c l 1$ marker was more than twice that observed in the two homozygous groups (Tremblay et al. 2004). The statistical significance of the differences was essentially unchanged after adjusting for changes in participation in vigorous physical activity as well as for changes in lipid and alcohol intake. These results confirm previous observations that there are interactions between genetic variation in the glucocorticoid receptor gene and ageing or undefined environmental factors. If further research confirms this finding, polymorphisms in the glucocorticoid receptor gene could be useful in the estimation of the 
(A)

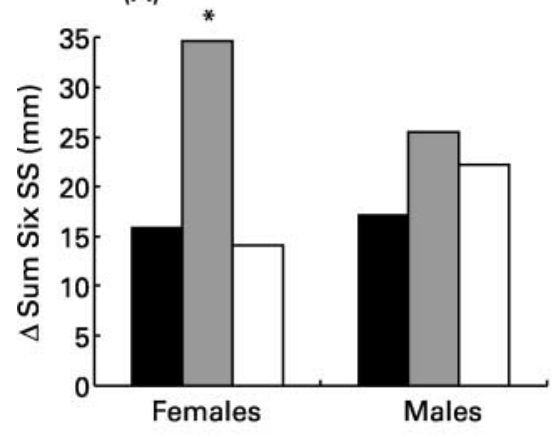

(B)

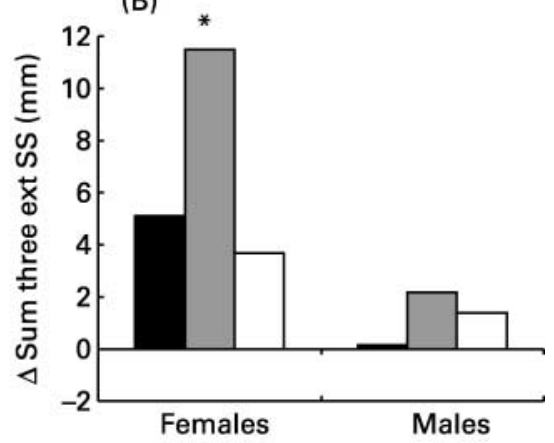

(C)

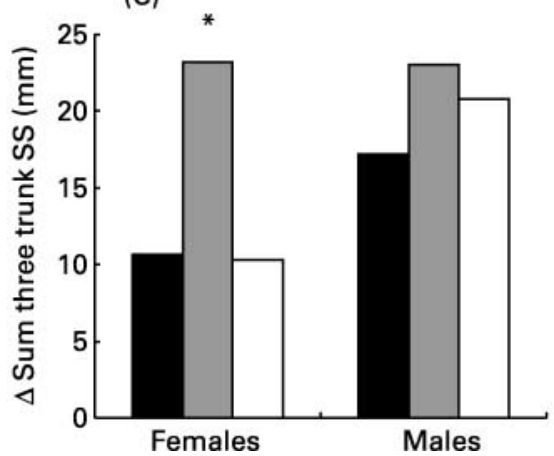

Fig. 1. Body-fat gain in young female subjects classified by genotype at the glucocorticoid receptor polymorphism of GRL IVS2-BC/1 RFLP. SS,; ext,. $\square, 4.5 / 4.5 ; \square, 4.5 / 2 \cdot 3 ; \square, 2 \cdot 3 / 2 \cdot 3$. Mean values were significantly different from those of the other two female groups: ${ }^{*} P<0.01$. (Reprinted with permission from Tremblay et al. 2004.)

risk of gaining substantial amounts of body fat with age or when challenged by chronic positive energy balance conditions.

\section{Summary}

In summary, experimental studies conducted with identical twins support the concept of gene-environment interaction effects on body fatness. These effects are of clinical relevance since they refer to genetically determined susceptibilities towards obesity that characterize some individuals. In this regard, the glucocorticoid receptor gene is emerging as a candidate that may be involved in a variety of interactive effects.

\section{Acknowledgements}

C. B. is funded in part by the George A. Bray Chair in Nutrition. A. T. is funded in part by the Canada Research Chair in Physical Activity, Nutrition and Energy Balance.

\section{References}

Bouchard A, Tremblay A, Després J-P, Thériault G, Nadeau A, Lupien PJ, Moorjani S, Prud'homme D \& Fournier G (1994) The response to exercise with constant energy intake in identical twins. Obes Res 2, 400-410.

Bouchard C, Tremblay A, Despres JP, Nadeau A, Lupien PJ, Theriault G, Dussault J, Moorjani S, Pinault S \& Fournier G (1990) The response to long-term overfeeding in identical twins. New Engl J Med 322, 1477-1482.
Buemann B, Vohl MC, Chagnon M, et al. (1997) Abdominal visceral fat is associated with a BclI restriction fragment length polymorphism at the glucocorticoid receptor gene locus. Obes Res 5, 186-192.

Chagnon YC, Rankinen T, Snyder EE, Weisnagel SJ, Perusse L \& Bouchard C (2003) The human obesity gene map: the 2002 update. Obes Res 11, 313-367.

Doucet E, Imbeault P, St-Pierre S, Almeras N, Mauriege P, Richard D \& Tremblay A (2000) Appetite after weight loss by energy restriction and a low-fat diet-exercise follow-up. Int J Obes 24, 906-914.

Lawton CL, Burley VJ, Wales JK \& Blundell JE (1993) Dietary fat and appetite control in obese subjects: weak effects on satiety. Int J Obes 17, 409-416.

Poehlman ET, Despres JP, Marcotte M, Tremblay A, Theriault G \& Bouchard C (1986a) Genotype dependency of adaptation in adipose tissue metabolism after short-term overfeeding. Am J Physiol 250, E480-E485.

Poehlman ET, Tremblay A, Fontaine E, Despres JP, Nadeau A, Dussault J \& Bouchard C (1986b) Genotype dependency of the thermic effect of a meal and associated hormonal changes following short-term overfeeding. Metabolism 35, $30-36$.

Poehlman ET, Tremblay A, Marcotte M, Pérusse L, Thériault G \& Bouchard C (1987) Heredity and changes in body composition and adipose tissue metabolism after short-term exercise-training. Eur J Appl Physiol 56, 398-402.

Poehlman ET, Tremblay A, Nadeau A, Dussault J, Thériault G \& Bouchard C (1986c) Heredity and changes in hormones and metabolic rates with short-term training. Am J Physiol 250, E711-E717.

Romsos DR, Van der Tuig JG, Kerner J \& Grogan CK (1987) Energy balance in rats with obesity-producing hypothalamic knife cuts: effects of adrenalectomy. J Nutr 117, 1121-1128. 
Rosmond R, Chagnon YC, Chagnon M, Perusse L, Bouchard C \& Bjorntorp P (2000) A polymorphism of the $5^{\prime}$-flanking region of the glucocorticoid receptor gene locus is associated with basal cortisol secretion in men. Metabolism 49, 1197-1199.

Tokunaga K, Fukushima M, Lupien JR, Bray GA, Kemnitz JW \& Schemmel R (1989) Effects of food restriction and adrenalectomy in rats with VMH or PVH lesions. Physiol Behav 45, $1131-1137$.

Tremblay A, Bouchard L, Bouchard C, Després JP, Drapeau V \& Pérusse L (2004) Long-term adiposity changes are related to a glucocorticoid receptor polymorphism in young females. J Clin Endocrinol Metab (In Press).

Tremblay A, Despres JP, Theriault G, Fournier G \& Bouchard C (1992) Overfeeding and energy expenditure in humans. Am J Clin Nutr 56, 857-862.
Tremblay A, Plourde G, Després JP \& Bouchard C (1989) Impact of dietary fat content and fat oxidation on energy intake in humans. Am J Clin Nutr 49, 799-805.

Tremblay A, Poehlman ET, Després J-P, Thériault G, Danforth E \& Bouchard C (1997) Endurance training with constant energy intake in identical twins: changes over time in energy expenditure and related hormones. Metabolism 46, 499-503.

Tremblay A, Poehlman ET, Nadeau A, Dussault J \& Bouchard C (1987) Heredity and overfeeding-induced changes in submaximal exercise $\mathrm{VO}_{2}$. J Appl Physiol 62, 539-544.

Ukkola O \& Bouchard C (2004) Role of candidate genes in the responses to long-term overfeeding: review of findings. Obes Rev (In Press).

World Health Organization (1998) Preventing and Managing the Global Epidemic. Geneva: WHO. 